\title{
Inhibition of histone deacetylase 2 mitigates profibrotic TGF- $\beta 1$ responses in fibroblasts derived from Peyronie's plaque
}

\author{
Ji-Kan Ryu, Woo-Jean Kim, Min-Ji Choi, Jin-Mi Park, Kang-Moon Song, Mi-Hye Kwon, Nando-Dulal Das, \\ Ki-Dong Kwon, Dulguun Batbold, Guo-Nan Yin and Jun-Kyu Suh
}

Epigenetic modifications, such as histone acetylation/deacetylation, have been shown to play a role in the pathogenesis of fibrotic disease. Peyronie's disease (PD) is a localized fibrotic process of the tunica albuginea, which leads to penile deformity. This study was undertaken to determine the anti-fibrotic effect of small interfering RNA (siRNA)-mediated silencing of histone deacetylase 2 (HDAC2) in primary fibroblasts derived from human PD plaque. PD fibroblasts were pre-treated with HDAC2 siRNA and then stimulated with transforming growth factor- $\beta 1$ (TGF- $\beta 1$ ). Protein was extracted from treated fibroblasts for Western blotting and the membranes were probed with antibody to phospho-Smad2/Smad2, phospho-Smad3/Smad3, smooth muscle $\alpha$-actin and extracellular matrix proteins, including plasminogen activator inhibitor-1, fibronectin, collagen I and collagen IV. We also performed immunocytochemistry to detect the expression of extracellular matrix proteins and to examine the effect of HDAC2 siRNA on the TGF- $\beta 1$-induced nuclear translocation of Smad2/3 in fibroblasts. Knockdown of HDAC2 in PD fibroblasts abrogated TGF- $\beta 1$-induced extracellular matrix production by blocking TGF- $\beta 1$-induced phosphorylation and nuclear translocation of Smad2 and Smad3, and by inhibiting TGF- $\beta 1$-induced transdifferentiation of fibroblasts into myofibroblasts. Decoding the individual function of the HDAC isoforms by use of siRNA technology, preferably siRNA for HDAC2, may lead to the development of specific and safe epigenetic therapies for PD.

Asian Journal of Andrology (2013) 15, 640-645; doi:10.1038/aja.2013.61; published online 17 June 2013

Keywords: cell culture; fibrosis; histone deacetylase; Peyronie's disease; transforming growth factor-beta

\section{INTRODUCTION}

Peyronie's disease (PD) is a localized fibrotic process of the tunica albuginea (TA), an envelope that covers erectile tissue, which leads to penile deformity and curvature and sometimes to erectile dysfunction. ${ }^{1,2}$ The molecular mechanisms responsible for chronic and progressive fibrosis are not yet completely delineated, and the medical treatments currently available fail to alter the progression of PD. So far, surgical intervention is the only efficacious treatment for PD. ${ }^{3,4}$ Therefore, along with an increased understanding of $\mathrm{PD}$ at the molecular level, the identification of the molecular target involved in the fibrogenic process is needed to develop novel medical treatments for PD.

Several profibrotic cytokines have been shown to be involved in the pathogenesis of PD. ${ }^{5}$ Accumulating evidence suggests that transforming growth factor- $\beta 1$ (TGF- $\beta 1$ ) is one of the most relevant fibrogenic cytokines associated with PD. ${ }^{6-8}$ The expression and activity of TGF$\beta 1$ and its downstream signaling cascade, such as Smad2 and Smad3 transcription factors, have been shown to be increased in human PD plaque. ${ }^{7,8}$ Moreover, we recently reported that a small-molecule inhibitor of activin receptor-like kinase 5, a TGF- $\beta$ type I receptor, attenuated fibrotic responses in both a rat model of $\mathrm{PD}$ in vivo ${ }^{9}$ and in fibroblasts isolated from human PD plaque in vitro. ${ }^{8}$
Epigenetic modifications, such as histone acetylation/deacetylation, have been shown to play a role in both the pathogenesis of fibrotic disease and inflammatory disorders. ${ }^{10,11}$ Histone deacetylases (HDACs) are classified into four groups on the basis of their similarity to yeast HDACs: class I (HDACs 1, 2, 3 and 8), class II (HDACs 4, 5, 6, 7, 9 and 10), class III (sirtuins 1-7) and class IV (HDAC11). ${ }^{12}$ Recent studies have demonstrated that HDACs are involved in tissue fibrosis in various organs, including urinary bladder, kidney, liver, heart and lung. ${ }^{10,13,14}$ In addition, small-molecule inhibitors of HDAC abrogate the activation and proliferation of fibroblasts in vitro and lessen tissue fibrosis in vivo. ${ }^{10,13}$ Although the exact mechanisms by which HDAC mediates fibrotic responses remain largely unknown, HDAC inhibitors are known to repress the TGF- $\beta$ pathway. ${ }^{15}$ However, none of the currently available HDAC inhibitors is specific for a single isoform of HDAC, which increases toxicity and limits the clinical applications. A previous study reported that HDAC2 activity was increased in the kidneys of diabetic rats and TGF- $\beta 1$-stimulated rat kidney tubular epithelial cells, and that knockdown of HDAC2 with small interfering RNA (siRNA) decreased fibronectin expression in kidney tubular epithelial cells. ${ }^{16}$ However, little is known as yet about the regulation and function of HDACs in PD. 
In the present study, therefore, we determined the effectiveness of the knockdown of HDAC2 on the TGF- $\beta 1$-induced profibrotic responses in primary fibroblasts derived from human $\mathrm{PD}$ plaque.

\section{MATERIALS AND METHODS}

\section{Primary fibroblast culture}

After obtaining informed consent, the plaque tissues were isolated from patients with PD who underwent surgical correction. The procedures were approved by the internal review board of our university. The tissue samples were used for primary fibroblast culture as previously described. ${ }^{8}$ Briefly, the plaque tissue was transferred into sterile vials containing Hank's balanced salt solution (Gibco, Carlsbad, CA, USA) and was washed three times in phosphate-buffered saline. Biopsy tissue was minced into $1-\mathrm{mm}^{2}$ segments and incubated in a shaker in $12.5 \mathrm{ml}$ Dulbecco's modified Eagle medium supplemented with $0.06 \%$ collagenase A (Sigma-Aldrich, St Louis, MO, USA) for $1 \mathrm{~h}$. The cells and tissue fragments were collected by centrifugation (400 $\mathrm{g} \times 5 \mathrm{~min}$ ), washed in fresh culture medium, and placed in 100$\mathrm{mm}$ cell culture dishes (Falcon-Becton Dickinson Labware, Franklin Lakes, NJ, USA) under standard cell culture conditions with Dulbecco's modified Eagle medium supplemented with $10 \%$ fetal calf serum, penicillin $\left(100 \mathrm{U} \mathrm{ml}^{-1}\right)$ and streptomycin $\left(100 \mu \mathrm{g} \mathrm{ml}^{-1}\right)$. The dishes were incubated in a humidified $37{ }^{\circ} \mathrm{C}$ incubator with $5 \% \mathrm{CO}_{2}$. The cells were then characterized as previously described. ${ }^{8}$ Passages five to eight were used for experimentation. We used four cell lines in this study.

\section{Transfection of siRNA into cells}

The fibroblasts were serum-starved for $24 \mathrm{~h}$ and transfected with 100 pmol siRNA oligonucleotides targeted specifically to HDAC2 (Santa Cruz Biotechnology Inc., Delaware, CA, USA) by using Lipofectamine 2000 (Gibco). In parallel, 100 pmol scramble siRNA was used as a control. After transfection, cells were plated and cultured for $48 \mathrm{~h}$ in Dulbecco's modified Eagle medium. The fibroblasts were then treated with $10 \mathrm{ng} \mathrm{ml} \mathrm{m}^{-1}$ TGF- $\beta 1$ (R\&D Systems Inc., Minneapolis, MN, USA) for $24 \mathrm{~h}$ to detect the protein expression of plasminogen activator inhibitor-1 (PAI-1), fibronectin, collagen subtypes, smooth muscle $\alpha$-actin and HDAC2, or were treated with TGF- $\beta 1$ for $1 \mathrm{~h}$ to detect the protein expression of phospho-Smad2 (PSmad2), phospho-Smad3 (P-Smad3) and total Smad2/3.

\section{RT-PCR}

Human HDAC2 mRNA expression was determined by semi-quantitative RT-PCR. Briefly, total RNA was extracted from cultured cells with Trizol (Invitrogen, Carlsbad, CA, USA) according to the manufacturer's protocols. RNA was reverse-transcribed by use of the Reverse Transcription System (Promega, Madison, WI, USA) according to the manufacturer's instructions. RT-PCR fragments were amplified by using the AccuPower PCR premix (Bioneer, Alameda, CA, USA). The PCR reaction was performed with denaturation at $94{ }^{\circ} \mathrm{C}$ for $1 \mathrm{~min}$, annealing at $60{ }^{\circ} \mathrm{C}$ for $30 \mathrm{~s}$, and extension at $72{ }^{\circ} \mathrm{C}$ for $30 \mathrm{~s}(25$ cycles). For the analysis of PCR products, $10 \mu \mathrm{l}$ of each PCR reaction was electrophoresed on a $1 \%$ agarose gel and DNA bands were visualized with a UV illuminator (Image Station IS4000R system; Kodak, Rochester, NY, USA). GAPDH was used as an internal control. The following primer pairs were used: human $H D A C 2,5^{\prime}$-CAT CCC ATG AAG CCT CAT AGA ATC-3' (forward), 5' -GCA CCA ATA TCC CTC AAG TCT CC-3' (reverse); and GAPDH, 5' ${ }^{\prime}$ CCA CTG GCG TCT TCA CCA C-3' (forward), 5' -CCT GCT TCA CCA CCT TCT TG-3' (reverse).

\section{Western blot}

Equal amounts of protein from whole-cell extracts (50 $\mu \mathrm{g}$ per lane) were electrophoresed on $12 \%$ sodium dodecylsulfate-polyacrylamide gels, transferred to nitrocellulose membranes, and probed with antibody against HDAC2 (Santa Cruz Biotechnology, Santa Cruz, CA, USA; $1: 100$ ), PAI-1 (Abcam, Cambridge, UK; 1:300), fibronectin (Abcam; 1:300), collagen I (Abcam; 1:300), collagen IV (Abcam; $1: 300$ ), smooth muscle $\alpha$-actin (Sigma-Aldrich; $1: 300$ ), p-Smad2 (s465/467, Cell signaling, Beverly, MA, USA; $1: 200$ ), p-Smad3 (s423/425, Cell signaling; $1: 200$ ), Smad2/3 (Cell signaling; $1: 200$ ) or $\beta$-actin (Abcam; $1: 6000$ ). Results were quantified by densitometry.

\section{Fluorescent immunocytochemistry}

The fibroblasts were cultured on sterile cover glasses (Marienfeld Laboratory, Lauda-Königshofen, Germany) and grown until nearly confluent. The cells were washed three times with phosphate-buffered saline and then fixed in $4 \%$ paraformaldehyde for $10 \mathrm{~min}$ at $4{ }^{\circ} \mathrm{C}$ and in $100 \%$ methanol for $10 \mathrm{~min}$ at $4{ }^{\circ} \mathrm{C}$. Individual chambers were incubated with antibody to HDAC2 (Santa Cruz Biotechnology; $1: 100$ ), PAI-1 (Abcam; 1:300), fibronectin (Abcam; 1:300), collagen I (Abcam; 1:300), collagen IV (Abcam; $1: 300$ ), smooth muscle $\alpha$-actin (Sigma-Aldrich; $1: 300$ ) or Smad2/3 (Cell signaling; $1: 200$ ) overnight at $4{ }^{\circ} \mathrm{C}$ in a moist chamber. After several washes with phosphatebuffered saline, the chambers were incubated with fluorescein isothiocyanate-conjugated (Zymed Laboratories, South San Francisco, CA, USA; 1:300) or tetramethyl rhodamine isothiocyanate-conjugated (Jackson ImmunoResearch Laboratories Inc., West Grove, PA, USA; $1: 300)$ secondary antibodies for $3 \mathrm{~h}$ at room temperature. Mounting medium containing 4,6-diamidino-2-phenylindole (DAPI; Vector Laboratories Inc., Burlingame, CA, USA) was applied to the chamber and nuclei were labeled. Signals were visualized, and digital images were obtained with a confocal microscope (FV1000; Olympus, Tokyo, Japan) under identical exposure settings.

\section{Statistical analysis}

Results are expressed as means \pm s.e. The group comparisons of parametric data were made by one-way analysis of variance followed by Newman-Keuls post hoc tests. We used the Kruskal-Wallis tests for nonparametric data. We performed statistical analysis with SigmaStat 3.5 software (Systat Software Inc., Richmond, CA, USA). We tested data for normality and variance. $P$ values less than 0.05 were considered significant.

\section{RESULTS}

HDAC2 knockdown inhibits extracellular matrix production induced by TGF- $\beta 1$ in fibroblasts derived from human PD plaque To determine the role of HDAC2 in extracellular matrix production, the siRNA approach was used. PD fibroblasts were transfected with siRNA specifically targeting HDAC2. RT-PCR analysis revealed that HDAC2 expression was inhibited by $60 \%$ in cells transfected with HDAC2 siRNA compared with the expression in cells transfected with scramble siRNA $(P<0.01$ by ANOVA; Figure 1a). The treatment of PD fibroblasts with TGF- $\beta 1$-induced HDAC2 protein expression, which returned to the baseline level after treatment with HDAC2 siRNA $(P<0.05$ by ANOVA; Figure $\mathbf{1} \mathbf{b}$ and $2 \mathbf{a})$. Both Western blot analysis and fluorescent immunocytochemistry showed that HDAC2 siRNA profoundly inhibited TGF- $\beta 1$-induced production of PAI- 1 , fibronectin, collagen I, and collagen IV in PD fibroblasts $(P<0.01$ for PAI- 1 and $P<0.05$ for fibronectin, collagen I and collagen IV by Kruskal-Wallis tests; Figure 1c and 2b). 
a

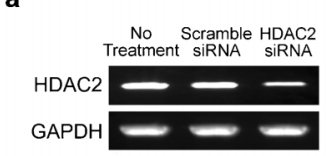

b

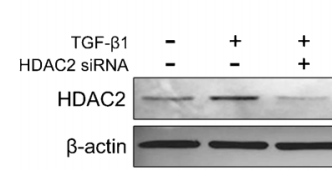

c
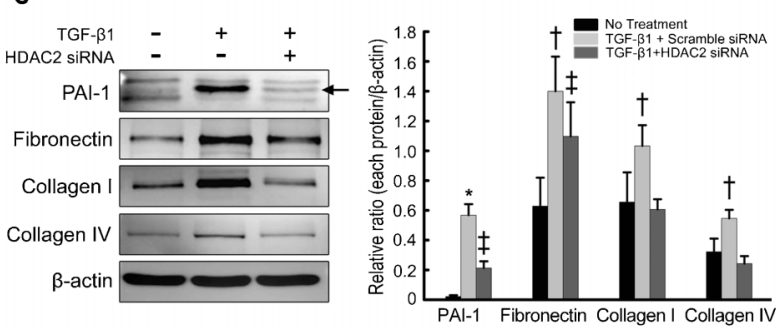

Figure 1 siRNA-mediated silencing of HDAC2 inhibits TGF- $\beta 1$-induced extracellular matrix protein production in fibroblasts derived from human PD plaque. (a) Expression of mRNA for HDAC2 in PD fibroblasts after specific knockdown using siRNA or control siRNA (scramble siRNA). Data are presented as the ratio of the product of HDAC2 gene transcript to that of GAPDH mRNA. Each bar depicts the mean values $( \pm$ s.e.) from four experiments per group. The relative ratio measured in the no treatment group was arbitrary presented as $1 . * P<0.01$ compared with the no treatment and scramble siRNA groups by ANOVA. (b) Effect of TGF- $\beta 1$ on HDAC2 protein expression. Fibroblasts were transfected with scramble siRNA or siRNA specific to HDAC2 by using Lipofectamine reagent for $48 \mathrm{~h}$ and were then treated with TGF- $\beta 1\left(10 \mathrm{ng} \mathrm{ml}^{-1}\right)$ for $24 \mathrm{~h}$. Whole-cell extracts were fractionated in a sodium dodecylsulfate-polyacrylamide gel. Data are presented as the relative density of HDAC2 protein compared with that of $\beta$-actin. Each bar depicts the mean values ( \pm s.e.) from four experiments per group. The relative ratio measured in the no treatment group was arbitrary presented as 1 . $* P<0.05$ compared with no treatment group, ${ }^{\dagger} P<0.05$ compared with TGF$\beta 1+$ the scramble siRNA group by ANOVA. (c) Representative Western blot for PAI-1, fibronectin, collagen I, and collagen IV in fibroblasts. Data are presented as the relative density of each protein compared with that of $\beta$-actin. Each bar depicts the mean values $( \pm$ s.e.) from four experiments per group. $* P<0.01$, ${ }^{\dagger} P<0.05$ compared with other groups, ${ }^{\ddagger} P<0.05$ compared with no treatment group by Kruskal-Wallis tests. HDAC2, histone deacetylase 2; PAI-1, plasminogen activator inhibitor-1; PD, Peyronie's disease; siRNA, small interfering RNA; TGF- $\beta 1$, transforming growth factor- $\beta 1$.

HDAC2 knockdown inhibits TGF- $\beta 1$-induced myofibroblastic differentiation in fibroblasts derived from human PD plaque To examine the molecular link between TGF- $\beta 1$-induced myofibroblastic differentiation and HDAC activity, PD fibroblasts were treated with HDAC2 siRNA. The expression of smooth muscle $\alpha$-actin, a marker for myofibroblasts, at the protein level was determined with Western blot analysis. The treatment of PD fibroblasts with TGF- $\beta 1$ resulted in an increase in smooth muscle $\alpha$-actin expression, which was attenuated after treatment with HDAC2 siRNA $(P<0.05$ by ANOVA; Figure $3 \mathbf{a}$ and $\mathbf{b}$ ). This finding suggests that HDAC2 activity is required for TGF- $\beta 1$-mediated myofibroblastic differentiation.

To determine the effect of knockdown of HDAC2 on TGF- $\beta 1$ induced morphologic changes in PD fibroblasts, we also performed a
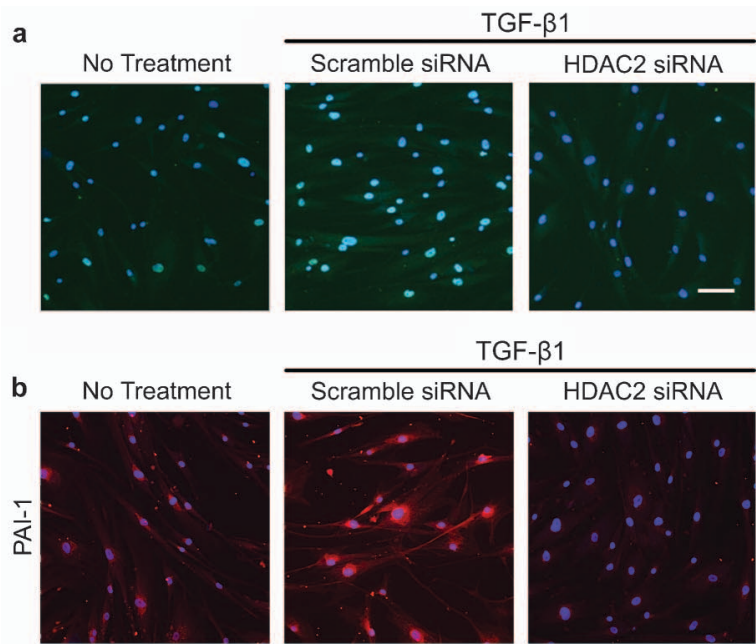

TGF- $\beta 1$
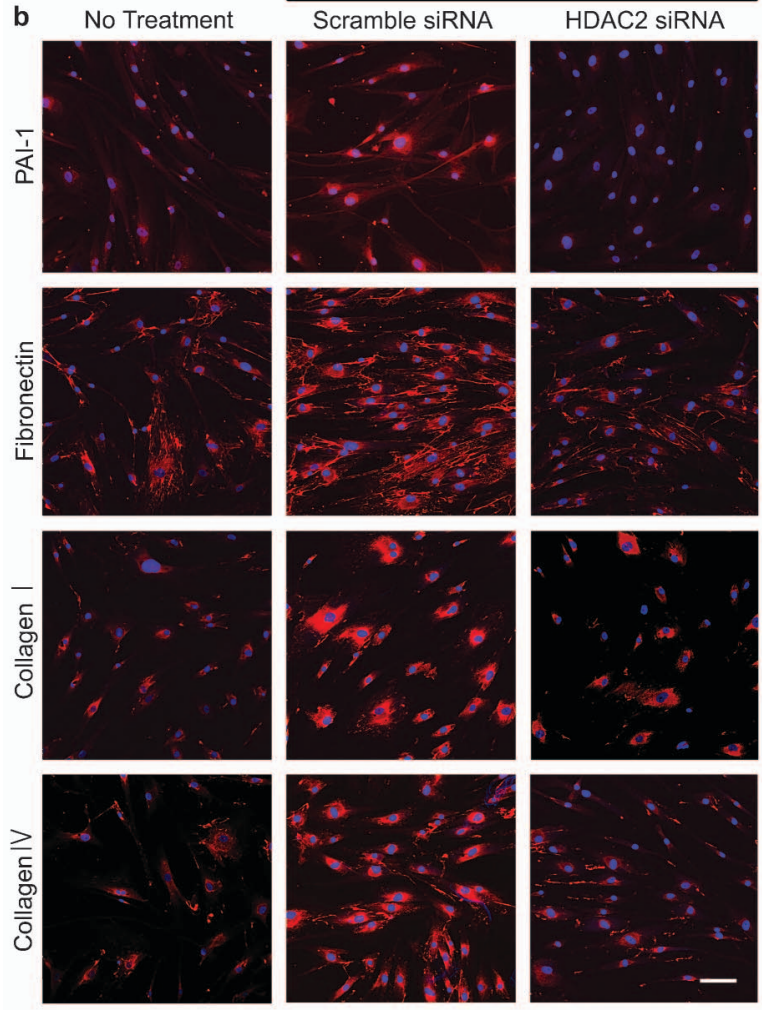

Figure 2 Fluorescent immunocytochemistry showing the inhibition of TGF- $\beta 1$ induced extracellular matrix protein expression by HDAC2 siRNA in fibroblasts derived from human PD plaque. (a) Representative fluorescent immunocytochemistry of fibroblasts with antibody against HDAC2. Nuclei were labeled with the DNA dye DAPI. Bar indicates $50 \mu \mathrm{m}$. Results were similar from four independent experiments. (b) Representative fluorescent immunocytochemistry of fibroblasts with antibody against PAI-1, fibronectin, collagen I and collagen IV. Nuclei were labeled with the DNA dye DAPI. Bar indicates $100 \mu \mathrm{m}$. Fibroblasts were transfected with scramble siRNA or siRNA specific to HDAC2 by using Lipofectamine reagent for $48 \mathrm{~h}$ and were then treated with TGF- $\beta 1$ (10 ng $\mathrm{ml}^{-1}$ ) for $24 \mathrm{~h}$. Results were similar from four independent experiments. DAPI, 4,6-diamidino-2-phenylindole; HDAC2, histone deacetylase 2; PAI-1, plasminogen activator inhibitor-1; PD, Peyronie's disease; siRNA, small interfering RNA; TGF- $\beta 1$, transforming growth factor- $\beta 1$.

fluorescent immunocytochemistry. HDAC2 siRNA successfully mitigated TGF- $\beta 1$-promoted $\alpha$-actin fiber formation (Figure $3 \mathrm{c}$ ).

HDAC2 knockdown inhibits Smad2 and Smad3 phosphorylation induced by TGF- $\beta 1$ in fibroblasts derived from human PD plaque A high level of phosphorylated Smad2 and Smad3 was detected in PD fibroblasts in response to a $1-\mathrm{h}$ treatment with TGF- $\beta 1$. The Smad2 and Smad3 phosphorylation was substantially inhibited by preincubation with HDAC2 siRNA $(P<0.01$ for phospho-Smad2 and $P<0.05$ for phospho-Smad3 by Kruskal-Wallis tests; Figure $4 \mathbf{a}$ and $\mathbf{b}$ ). 
a
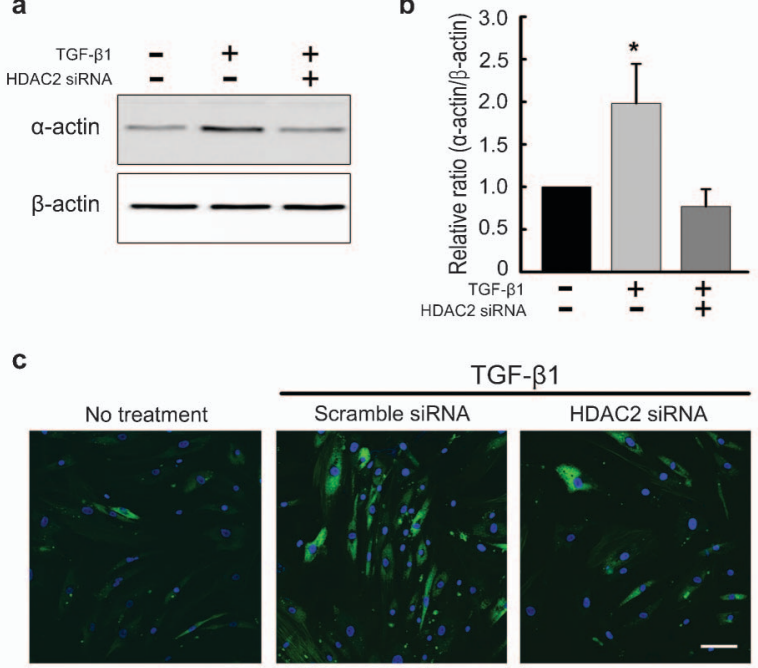

Figure 3 siRNA-mediated silencing of HDAC2 inhibits TGF- $\beta 1$-induced myofibroblastic differentiation in fibroblasts derived from human PD plaque. (a) Representative Western blot for smooth muscle $\alpha$-actin in fibroblasts. Fibroblasts were transfected with scramble siRNA or siRNA specific to HDAC2 by using Lipofectamine reagent for $48 \mathrm{~h}$ and were then treated with TGF- $\beta 1$ (10 ng $\mathrm{ml}^{-1}$ ) for $24 \mathrm{~h}$. Whole-cell extracts were fractionated in a sodium dodecylsulfatepolyacrylamide gel. (b) Data are presented as the relative density of smooth muscle $\alpha$-actin protein compared with that of $\beta$-actin. Each bar depicts the mean values ( \pm s.e.) from four experiments per group. The relative ratio measured in the no treatment group was arbitrary presented as 1 . $* P<0.05$ compared with the other groups by ANOVA. (c) Representative fluorescent immunocytochemistry of fibroblasts with antibody against smooth muscle $\alpha$-actin. Nuclei were labeled with the DNA dye DAPI. Bar indicates $100 \mu \mathrm{m}$. Results were similar from four independent experiments. DAPI, 4,6-diamidino-2-phenylindole; HDAC2, histone deacetylase 2; PD, Peyronie's disease; siRNA, small interfering RNA; TGF- $\beta 1$, transforming growth factor- $\beta 1$.

\section{HDAC2 knockdown efficiently inhibits nuclear translocation of} Smad $2 / 3$ induced by TGF- $\beta 1$ in fibroblasts derived from human PD plaque

TGF- $\beta 1$ has been shown to induce the translocation of Smad proteins from the cytoplasm to the nucleus. ${ }^{17}$ To evaluate whether HDAC2 activity is required for TGF- $\beta 1$-induced nuclear translocation of $\operatorname{Smad} 2 / 3$, we performed immunofluorescent staining of fibroblasts with antibody against total Smad2/3. HDAC2 siRNA reduced TGF$\beta 1$-induced nuclear accumulation of Smad proteins $(P<0.01$ by ANOVA; Figure 4c and d).

\section{DISCUSSION}

This study demonstrated that knockdown of HDAC2 significantly decreased the TGF- $\beta 1$-induced accumulation of extracellular matrix in human PD fibroblasts by blocking TGF- $\beta$ signaling, i.e., the phosphorylation and nuclear shuttling of Smad 2 and Smad3, and by inhibiting TGF- $\beta 1$-induced myofibroblastic differentiation.

Epigenetic modifications refer to heritable changes in gene activity over rounds of cell division without changes in DNA sequence. In previous studies, an increase in HDAC1 and HDAC2 expression was observed in fibrotic kidney induced by unilateral ureteral obstruction, ${ }^{18}$ and HDAC2 siRNA revealed anti-fibrotic effects in vitro. ${ }^{16}$ Recent studies have also reported epigenetic modifications in gene transcription in systemic sclerosis, ${ }^{19,20}$ a disease characterized by severe fibrosis of the skin and various internal organs, and silencing of HDAC7 decreased the production of collagen I and collagen III. ${ }^{21}$ These findings suggest an
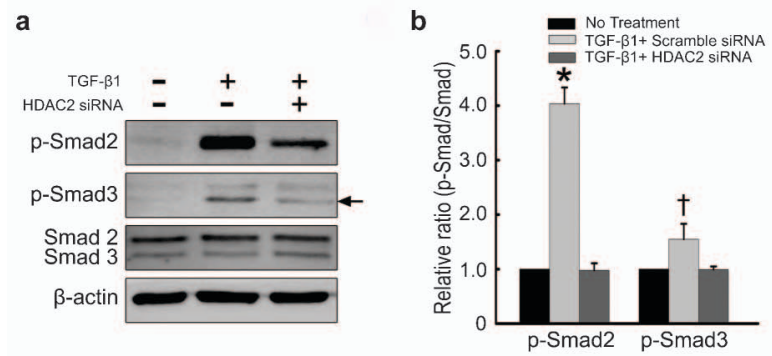

C
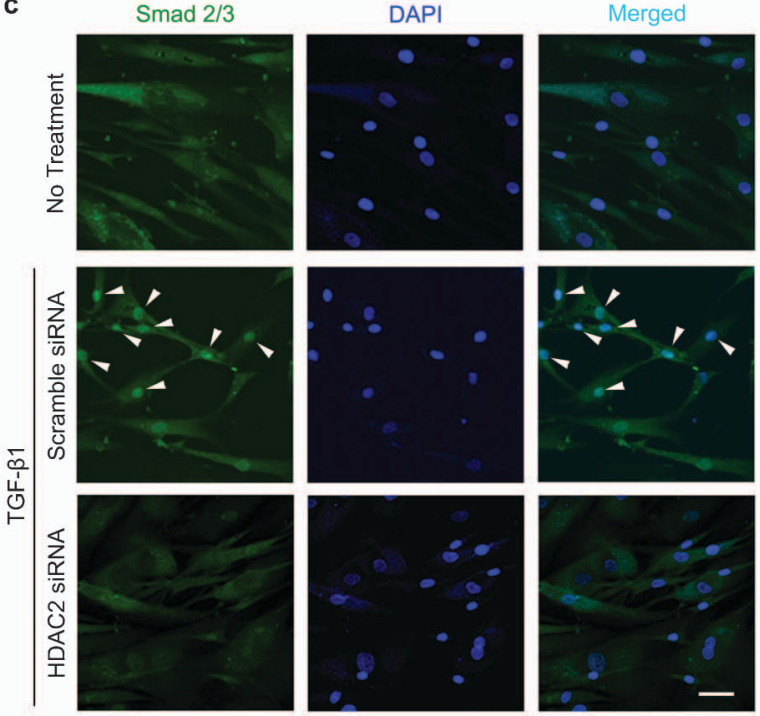

d

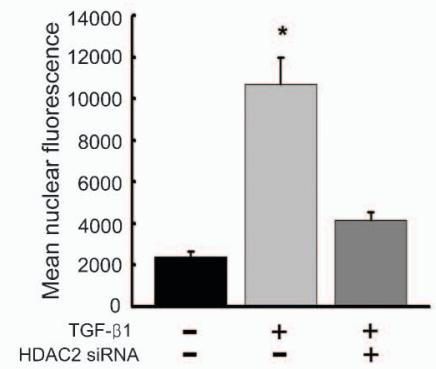

Figure 4 siRNA-mediated silencing of HDAC2 suppresses TGF- $\beta 1$-induced Smad2 and Smad3 phosphorylation and nuclear translocation in fibroblasts derived from human PD plaque. (a) Representative Western blot for phosphoSmad2 ( $p-S m a d 2)$, phospho-Smad3 ( $p-S m a d 3$ ), and total Smad2/3. Fibroblasts were transfected with scramble siRNA or siRNA specific to HDAC2 by using Lipofectamine reagent for $48 \mathrm{~h}$ and were then treated with TGF- $\beta 1\left(10 \mathrm{ng} \mathrm{ml}^{-1}\right.$ ) for $1 \mathrm{~h}$. Whole-cell extracts were fractionated in a sodium dodecylsulfate-polyacrylamide gel. Arrows denote target bands. (b) Data are presented as the ratio of phosphorylated protein to total protein. The relative ratio measured in the no treatment group was arbitrary presented as 1 . Each bar depicts the mean values ( \pm s.e.) from four experiments per group. ${ }^{*} P<0.01,{ }^{\dagger} P<0.05$ compared with the other groups by Kruskal-Wallis tests. (c) Representative fluorescent immunocytochemistry of primary human fibroblasts with antibody against total Smad2/3. Nuclei were labeled with the DNA dye DAPI. Arrow heads denote nuclear translocation of Smad2/3. Bar indicates $50 \mu \mathrm{m}$. (d) Nuclear fluorescence intensity was quantified for all cells. Each bar depicts the mean values ( \pm s.e.) from four experiments per group. $* P<0.01$ compared with the other groups by ANOVA. DAPI, 4,6diamidino-2-phenylindole; HDAC2, histone deacetylase 2; PD, Peyronie's disease; siRNA, small interfering RNA; TGF- $\beta 1$, transforming growth factor- $\beta 1$.

importance of epigenetic modification in the pathogenesis of fibrotic diseases and, furthermore, that therapies aimed at blocking individual HDAC isoforms might be efficacious for these conditions. 
In the present study, the cytokine (TGF- $\beta 1$ )-induced expression of HDAC2 was significantly increased in the PD fibroblasts. Knockdown of HDAC2 with its specific siRNA in PD fibroblasts significantly inhibited the TGF- $\beta 1$-induced production of PAI- 1 , fibronectin, collagen I and collagen IV proteins. A recent study reported that, among six HDACs tested (HDAC1-5 and HDAC8), HDAC2 activity was significantly increased in both the kidneys of diabetic rats and TGF- $\beta 1$ treated renal tubular epithelial cells. ${ }^{16}$ In addition, treatment with HDAC2 siRNA decreased the mRNA expression of fibronectin in TGF- $\beta 1$-stimulated renal tubular epithelial cells, ${ }^{16}$ which suggests a potent anti-fibrotic action of HDAC2 siRNA in a variety of fibrogenic processes.

Previous studies reported that increased HDAC activity is required for TGF- $\beta 1$-induced myofibroblastic differentiation. ${ }^{22-24}$ In the present study, treatment of PD fibroblasts with TGF- $\beta 1$ induced fibroblast-to-myofibroblast transition, as evidenced by both Western blot analysis and immunocytochemical staining for smooth muscle $\alpha$ actin. Similar to a previous study that showed a decrease in the mRNA expression of smooth muscle $\alpha$-actin in renal tubular epithelial cells when HDAC2 was knocked down, ${ }^{16}$ inhibition of HDAC2 abrogated TGF- $\beta 1$-induced transdifferentiation of PD fibroblasts into myofibroblasts. Moreover, inhibition of HDACs by trichostatin A, a nonspecific HDAC inhibitor, is known to decrease activation of myofibroblasts and expression of extracellular matrix in rat renal interstitial fibroblasts ${ }^{24}$ and in human skin fibroblasts exposed to TGF- $\beta 1{ }^{22}$ HDAC4 knockdown also inhibited TGF- $\beta 1$-stimulated smooth muscle $\alpha$-actin expression in normal human lung fibroblasts. ${ }^{23}$ Because the myofibroblast is the key effector cell type responsible for the production of extracellular matrix protein and subsequent fibrogenesis, unveiling the molecular events responsible for activation of PD fibroblasts may guide new approaches for halting the progression of chronic fibrotic processes in the TA.

Another mechanism by which knockdown of HDAC2 inhibits fibrotic responses in PD fibroblasts may be suppression of activation of the Smad transcriptional factors. The phosphorylation of Smad2 or Smad 3 is required for its nuclear accumulation and TGF- $\beta$ signaling, ${ }^{25}$ which is responsible for the deposition of extracellular matrix and tissue fibrosis. In the present study, silencing of HDAC2 prevented TGF- $\beta 1$-induced Smad 2 and Smad 3 phosphorylation and the nuclear accumulation of Smad proteins.

In the present study, we for the first time documented that silencing of HDAC2 has an anti-fibrotic effect in human PD fibroblasts in vitro. Although the individual HDAC isoforms have distinctive roles in both physiologic and pathologic conditions, most HDAC inhibitors target multiple isoforms and none of them can specifically block a single isoform of HDAC, which limits their therapeutic potential. For example, trichostatin A has been known to inhibit fibrotic responses in a variety of animal models or in cell culture systems. ${ }^{10,13,15,20}$ However, it was reported that trichostatin A also up-regulates the expression of connective tissue growth factor and intracellular adhesion molecule-1, which are responsible for the pathogenesis of systemic sclerosis. By contrast, silencing of HDAC7 demonstrated an anti-fibrotic effect without influencing the expression of these profibrotic molecules in fibroblasts isolated from patients with systemic sclerosis. ${ }^{21}$ Therefore, inhibition of individual HDAC isoforms by use of RNA interference technology may have advantages in the development of more specific and less toxic epigenetic therapies for PD. Further studies are needed to determine the therapeutic role of HDAC2 knockdown in PD models in vivo. A lack of experiments in fibroblasts derived from normal TA represents a limitation of this study.
In summary, knockdown of HDAC2 in PD fibroblasts abrogated TGF- $\beta 1$-induced extracellular matrix production and transdifferentiation of fibroblasts into myofibroblasts by blocking the TGF- $\beta 1$ induced activation of the Smad2/3 pathway. Decoding the individual function of the HDAC isoforms may open new avenues for developing specific and safe epigenetic therapies for PD.

\section{AUTHOR CONTRIBUTIONS}

J-KR participated in the design of the study, performed RT-PCR, and wrote the manuscript. WJK participated in the design of the study and analyzed the data, MJC and J-MP performed western blot and analyzed the data, K-MS and M-HK performed cell culture, NDD analyzed the data, K-DK, DB, and GNY performed immunocytochemical staining, J-KS participated in the design of the study and critically revised the manuscript for important intellectual content. All authors read and approved the final manuscript.

\section{COMPETING FINANCIAL INTERESTS}

The authors declare no competing financial interests.

\section{ACKNOWLEDGMENTS}

This study was supported by grant no. 2011-0015771 (Ji-Kan Ryu) from the Korea Science and Engineering Foundation funded by the Korea government (Ministry for Education, Science, and Technology) and by grant no. A110076 (Jun-Kyu Suh) from the Korea Healthcare technology R\&D Project, Ministry for Health, Welfare \& Family Affairs, Republic of Korea. We thank Jennifer Holmes for help in preparing the manuscript.

1 Hellstrom WJ, Bivalacqua TJ. Peyronie's disease: etiology, medical, and surgical therapy. J Androl 2000; 21: 347-54.

2 Gholami SS, Gonzalez-Cadavid NF, Lin CS, Rajfer J, Lue TF. Peyronie's disease: a review. J Urol 2003; 169: 1234-41.

3 Hauck EW, Diemer T, Schmelz HU, Weidner W. A critical analysis of nonsurgical treatment of Peyronie's disease. Eur Urol 2006; 49: 987-97.

4 Kadioglu A, Akman T, Sanli O, Gurkan L, Cakan M et al. Surgical treatment of Peyronie's disease: a critical analysis. Eur Urol 2006; 50: 235-48.

5 Gonzalez-Cadavid NF, Magee TR, Ferrini M, Qian A, Vernet D et al. Gene expression in Peyronie's disease. Int J Impot Res 2002; 14: 361-74.

6 El-Sakka AI, Hassoba HM, Pillarisetty RJ, Dahiya R, Lue TF. Peyronie's disease is associated with an increase in transforming growth factor-beta protein expression. J Urol 1997; 158: 1391-4.

7 Haag SM, Hauck EW, Szardening-Kirchner C, Diemer T, Cha ES et al. Alterations in the transforming growth factor (TGF)-beta pathway as a potential factor in the pathogenesis of Peyronie's disease. Eur Urol 2007; 51: 255-61.

8 Piao S, Choi MJ, Tumurbaatar M, Kim WJ, Jin HR et al. Transforming growth factor (TGF)- $\beta$ type I receptor kinase (ALK5) inhibitor alleviates profibrotic TGF- $\beta 1$ responses in fibroblasts derived from Peyronie's plaque. J Sex Med 2010; 7: 3385-95.

9 Ryu JK, Piao S, Shin HY, Choi MJ, Zhang LW et al. IN-1130, a novel transforming growth factor-beta type I receptor kinase (activin receptor-like kinase 5) inhibitor, promotes regression of fibrotic plaque and corrects penile curvature in a rat model of Peyronie's disease. J Sex Med 2009; 6: 1284-96.

10 Pang M, Zhuang S. Histone deacetylase: a potential therapeutic target for fibrotic disorders. J Pharmacol Exp Ther 2010; 335: 266-72.

11 McKinsey TA. Targeting inflammation in heart failure with histone deacetylase inhibitors. Mol Med 2011; 17: 434-41.

12 Gregoretti IV, Lee YM, Goodson HV. Molecular evolution of the histone deacetylase family: functional implications of phylogenetic analysis. J Mol Biol 2004; 338: 1731

13 Hodges SJ, Yoo JJ, Mishra N, Atala A. The effect of epigenetic therapy on congenital neurogenic bladders-a pilot study. Urology 2010; 75: 868-72.

14 Qin L, Han YP. Epigenetic repression of matrix metalloproteinases in myofibroblastic hepatic stellate cells through histone deacetylases 4 : implication in tissue fibrosis. Am J Pathol 2010; 177: 1915-28.

15 Rombouts K, Niki T, Greenwel P, Vandermonde A, Wielant A et al. Trichostatin A, a histone deacetylase inhibitor, suppresses collagen synthesis and prevents TGF-beta - $^{-}$ induced fibrogenesis in skin fibroblasts. Exp Cell Res 2002; 278: 184-97.

16 Noh H, Oh EY, Seo JY, Yu MR, Kim YO et al. Histone deacetylase-2 is a key regulator of diabetes- and transforming growth factor-betal-induced renal injury. Am J Physiol Renal Physiol 2009; 297: F729-39. 
17 Hoodless PA, Haerry T, Abdollah S, Stapleton M, O'Connor MB et al. MADR 1, a MADrelated protein that functions in BMP2 signaling pathways. Cell 1996; 85: 489-500.

18 Marumo T, Hishikawa K, Yoshikawa M, Hirahashi J, Kawachi S et al. Histone deacetylase modulates the pro-inflammatory and fibrotic changes in tubulointerstitial injury. Am J Physiol Renal Physiol 2009; 298: F133-41.

19 Ghosh AK, Varga J. The transcriptional coactivator and acetyltransferase p300 in fibroblast biology and fibrosis. J Cell Physiol 2007; 213: 663-71.

20 Huber LC, Distler JH, Moritz F, Hemmatazad H, Hauser T et al. Trichostatin A prevents the accumulation of extracellular matrix in a mouse model of bleomycin-induced skin fibrosis. Arthritis Rheum 2007; 56: 2755-64.

21 Hemmatazad H, Rodrigues HM, Maurer B, Brentano F, Pileckyte M et al. Histone deacetylase 7 , a potential target for the antifibrotic treatment of systemic sclerosis. Arthritis Rheum 2009; 60: 1519-29.
22 Glenisson W, Castronovo V, Waltregny D. Histone deacetylase 4 is required for TGFbeta1-induced myofibroblastic differentiation. Biochim Biophys Acta 2007; 1773: 1572-82.

23 Guo W, Shan B, Klingsberg RC, Qin X, Lasky JA. Abrogation of TGFbeta1-induced fibroblast-myofibroblast differentiation by histone deacetylase inhibition. $A m \mathrm{~J}$ Physiol Lung Cell Mol Physiol 2009; 297: L864-70.

24 Pang $\mathrm{M}$, Kothapally J, Mao H, Tolbert E, Ponnusamy $\mathrm{M}$ et al. Inhibition of his tone deacetylase activity attenuates renal fibroblast activation and interstitial fibrosis in obstructive nephropathy. Am J Physiol Renal Physiol 2009; 297: F9961005.

25 Macías-Silva M, Abdollah S, Hoodless PA, Pirone R, Attisano L et al. MADR2 is a substrate of the TGFbeta receptor and its phosphorylation is required for nuclear accumulation and signaling. Cell 1996; 87: 1215-24. 